\title{
NOTAS SOBRE ALGUNAS POLEMONIACEAE MEXICANAS ${ }^{1}$
}

\author{
Jerzy Rzedowski, Graciela Calderon de Rzedowski² \\ Instituto de Ecología \\ Centro Regional del Bajío \\ Apartado postal 386 \\ 61600 Pátzcuaro, Michoacán \\ Y \\ Jose A. Villarreal Q. \\ Departamento de Botánica \\ Universidad Autónoma Agraria "Antonio Narro" \\ 25315 Buenavista, Saltillo, Coahuila
}

\begin{abstract}
RESUMEN
Se proponen varios cambios pertinentes a la sistemática y nomenclatura de algunas especies mexicanas de Polemoniaceae.

Se considera a Bonplandia linearis Rob. sólo como una variante de B. geminiflora Cav. Igualmente Cobaea biaurita Standl. no es sino un sinónimo taxonómico de C. stipularis Benth. Loeselia greggii S. Wats. es el nombre correcto de la especie que se ha conocido como L. scariosa (Mart. \& Gal.) Walp. y se restituye el binomio L. pumila (Mart. \& Gal.) Walp. para la planta que fue erroneamente identificada como L. ramosissima (Mart. \& Gal.) Walp.

Con base en ejemplares colectados en Chihuahua, Durango, Guanajuato y Querétaro se describe como nuevo a Polemonium melindae, el cual difiere de $P$. pauciflorum $\mathrm{S}$. Wats. en sus corolas más anchas, más largas y de color amarillo claro, así como en los lóbulos del cáliz más anchos.
\end{abstract}

\footnotetext{
${ }^{1}$ Se agradece la colaboración recibida de la Dra. Alva Day, de la Academia de Ciencias de California, quien ayudó a despejar varias dudas. Se dan las gracias asimismo a la Dra. Lourdes Rico, de los Reales Jardines Botánicos de Kew, al Dr. Mauricio Velayo, del Real Jardín Botánico de Madrid, al Dr. Paul Bamps, del Jardín Botánico Nacional de Bélgica, a la M. en C. Ana Rosa López Ferrari y al M. en C. Adolfo Espejo, de la Universidad Autónoma Metropolitana, por haber buscado tipos de varios taxa de Polemoniaceae y proporcionado fotografías de los mismos. Las autoridades de los herbarios de la Universidad de Harvard no tuvieron inconveniente en enviar en préstamo dos ejemplares tipo. 2 Trabajo realizado con apoyo económico del Instituto de Ecología (cuenta 902-03), del Consejo Nacional de Ciencia y Tecnología y de la Comisión Nacional para el Conocimiento y Uso de la Biodiversidad.
} 


\section{ABSTRACT}

Several changes are proposed in the taxonomy and nomenclature of some Mexican members of the Polemoniaceae.

Bonplandia linearis Rob. is considered only as a minor variant of $B$. geminiflora Cav. Cobaea biaurita Standl. proves also to be a taxonomic synonym of $C$. stipularis Benth. Loeselia greggii $\mathrm{S}$. Wats. is the correct name of the species most often named L. scariosa (Mart. \& Gal.) Walp. and the name L. pumila (Mart. \& Gal.) is recognized for the plant erroneousely identified as L. ramosissima (Mart. \& Gal.) Walp.

Polemonium melindae is described as a new species on the basis of specimens collected in Chihuahua, Durango, Guanajuato and Querétaro. It differs from P. pauciflorum S. Wats. in its wider, longer and lemmon-colored corolla and in its wider calyx lobes.

La preparación del manuscrito de la familia Polemoniaceae para la Flora del Bajío y de Regiones Adyacentes ha tropezado con diversos obstáculos, pues a medida de ir profundizando nos encontramos con numerosos problemas tanto de la definición de las especies, como de la aplicación de nombres apropiados a las mismas. Como resultado de la revisión de un amplio material de herbario, de la observación de las poblaciones naturales en el campo, así como del examen de ejemplares tipo o de sus fotografías, se proponen las siguientes enmiendas y sugerencias.

\section{BONPLANDIA}

El género y el binomio B. geminiflora fueron publicados por Cavanilles en 1800 con fundamento en ejemplares obtenidos algunos años antes en Guerrero (L. Née s.n. (MA, foto en (EB)). No se registró adición alguna a Bonplandia durante todo el siglo XIX, pero en 1907 Robinson describió $B$. linearis con base en material colectado en Michoacán ( $C$. G. Pringle 10364, isotipo en MEXU!), señalando que su planta difiere de B. geminiflora en la forma de las hojas que son lineares o bien pinnatilobadas y con los lóbulos lineares, mientras que en la especie típica estas estructuras son mucho más anchas, a menudo ovadas.

El material de herbario que se tiene en la actualidad de $B$. geminiflora revela que es una especie particularmente variable en algunos caracteres, incluyendo el tamaño de la flor, el tipo y la abundancia de la pubescencia y sobre todo la forma y el tamaño de la hoja, sin que resulte factible diferenciar ni siquiera entidades infraespecíficas. En efecto, algunas poblaciones de Michoacán, de Jalisco y de Nayarit destacan notablemente por sus hojas o segmentos foliares muy angostos, pero con frecuencia en un solo individuo la anchura de estos órganos varía de manera extraordinaria. En consecuencia reducimos $B$. linearis a la sinonimia de $B$. geminiflora y restituimos al género la categoría de monotípico restringido en su distribución a México y Guatemala.

Standley (1924: 1213) consideró a estas plantas como sufrutescentes, pero como ya lo hizo notar Gibson (1970: 86), se trata de organismos anuales, aunque en ocasiones hasta de $2 \mathrm{~m}$ de alto. 


\section{COBAEA}

En su revisión de este género Standley (1914) describió y proporcionó una fotografía del tipo de $C$. biaurita, que es un ejemplar colectado por E. W. Nelson en Chiapas, indicando que la planta se distingue principalmente por sus foliolos basales más o menos profundamente auriculados, en conjunción con los segmentos del cáliz lanceolados. Nash (1979: 4) reconoció esta misma especie entre las polemoniáceas de Veracruz y mostró que se trata de una de las representantes más comunes de Cobaea que habitan en ese estado. Comentó asimismo que en algunos especímenes el par de foliolos inferiores está "raramente reducido y casi ovado".

En conexión con ello cabe advertir que la planta con foliolos basales muy reducidos y reniformes había sido descrita en 1840 por Bentham como C. stipularis, taxon cuyo tipo (K. T. Hartweg s.n. (K, foto en IEB)) procede del estado de Hidalgo.

El examen de numerosos ejemplares procedentes de Guanajuato, Querétaro, Hidalgo, Veracruz, Oaxaca y Chiapas revela que individuos con el aspecto de C. biaurita y con el aspecto de $C$. stipularis no son sino variantes de una sola especie y que por razones de prioridad cronológica este último es el nombre que debe usarse.

Al margen de lo anterior tampoco puede pasar inadvertida la gran similitud entre C. stipularis y C. scandens Cav. La segunda, a menudo llamada "hiedra morada" o "campana morada", al parecer sólo se conoce de plantas cultivadas o escapadas de cultivo y difiere primordialmente en sus segmentos del cáliz que son ovales a suborbiculares y con el ápice redondeado y apiculado.

Aunque se da la curiosa circunstancia de no haberse registrado para $C$. scandens ningún nombre común en náhuatl ni en alguna otra lengua indígena, no hay mucha duda de que esta planta es nativa de México y no sería remoto que $C$. scandens se hubiera originado como un mutante de $C$. stipularis y perpetuado por el hombre.

\section{GILIA}

En el NE de Guanajuato y en el NE de Querétaro se han colectado en repetidas ocasiones representantes de una especie del género Gilia, cuyas características coinciden bastante bien con las señaladas para G. incisa Benth. por Brand (1907: 147), por Wherry (1966: 294), así como con las proporcionadas e ilustradas por Nash (1979: 12).

Sin embargo, al revisar la descripción original de esta última especie nos llamaron la atención dos discrepancias. De acuerdo con Bentham (1845: 312), se trata de una planta "glaberrima" y las corolas miden "2 lin." (= ca. $4 \mathrm{~mm}$ ) de largo, mientras que en todos los ejemplares procedentes de nuestra área, al igual que en los demás identificados como $G$. incisa que pudimos encontrar en los principales herbarios mexicanos, los tallos son finamente glanduloso-pubescentes y la longitud de las flores varía entre (5)8 y 9(12) mm.

Por consiguiente, solicitamos para su examen el espécimen tipo ( $T$. Drummond coll. 3, No. 463), el cual en principio debería estar depositado en la colección del Real Jardín Botánico de Kew, pero no se le pudo hallar ahí ni tampoco en el Museo de Historia Natural de Londres. En fecha posterior logramos localizar un isotipo en el Herbario Gray de la 
Universidad de Harvard, mismo que nos ha sido prestado gentilmente. El ejemplar en cuestión, procedente de Texas, presenta la pubescencia característica en el tallo, pero carece de flores.

En virtud de lo anterior concluimos que, a pesar de las discrepancias con el protólogo, debe aceptarse el uso del nombre $G$. incisa para las plantas pubescentes y con corolas grandes.

\section{LOESELIA}

De las cinco especies de este género presentes en el área que abarca la Flora del Bajío y de Regiones Adyacentes dos requieren de enmiendas nomenclaturales.

La Dra. Alva Day, de la Academia de Ciencias de California, quien está preparando en la actualidad una revisión de Loeselia, encontró y comunicó que:

a) a la planta que desde los tiempos de la publicación de Brand (op. cit.) se ha estado llamando L. scariosa (Mart. \& Gal.) Walp. le corresponde el nombre de L. greggii S. Wats. (sintipos: J. Gregg $360(\mathrm{GH})$ y E. Palmer $1063(\mathrm{GH})$, fotocopia de ambos en IEB), ya que los sintipos de Hoitzia scariosa Mart. \& Gal. (H. Galeotti 638 y 1450 (BR, fotos en IEB)) no corresponden sino a una variante menor de $L$. coerulea (Cav.) G. Don (tipo: $L$. Née s.n. (MA, foto en IEB)).

b) Gibson (op. cit.: 95) y Nash (op. cit.: 23) utilizaron incorrectamente el binomio Loeselia ramosissima (Mart. \& Gal.) Walp. para la especie que debe denominarse L. pumila (Mart. \& Gal.) Walp. (tipo: H. Galeotti 7132 (BR, foto en IEB)). El material original de Hoitzia ramosissima Mart. \& Gal. (sintipos: H. Galeotti 1444 y 1453 (BR, fotos en IEB)) permite concluir que esta última es un sinónimo taxonómico de L. glandulosa (Cav.) G. Don (tipo: L. Née s.n. (MA, foto en IEB)).

Después de examinar las fotografías de los tipos de todos estos taxa, confirmamos plenamente las conclusiones de la Dra. Day.

\section{POLEMONIUM}

Colectas recientes realizadas en Querétaro y en Guanajuato han revelado la existencia de una especie de flores muy vistosas perteneciente a este género, que al parecer no ha sido descrita todavía. Una revisión del material depositado en varios herbarios indica que la planta en cuestión también se ha encontrado en Chihuahua y en Durango.

Polemonium melindae sp. n. (Fig. 1)

Planta procumbens vel ascendens, caules subglabri usque ad dense lanosi; foliola plerumque lanceolata, 7-25 mm longa, 2-6 mm lata; flores plerumque solitarii vel geminati; calyx 15-21(25) mm longus, lobis triangularibus, tubo 1-2-plo longioribus; corolla anguste infundibuliformis, citrina vel viridi-flava, $4.5-7 \mathrm{~cm}$ longa, ad tubi medium (7)8-11 $\mathrm{mm}$ lata, ad tubi apicem 1.5-3.5 cm lata, lobis suborbicularibus; stamina inclusa vel exserta, filamentis longitudine inaequalibus; capsula subcylindrica, 9-10 mm longa. 

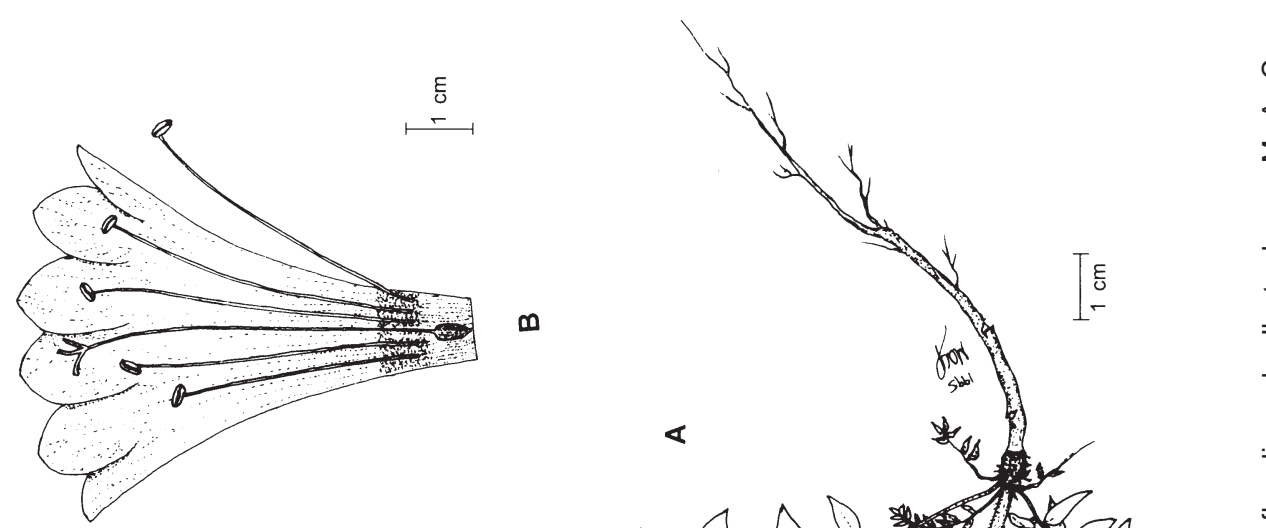

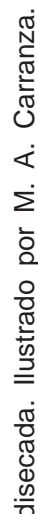
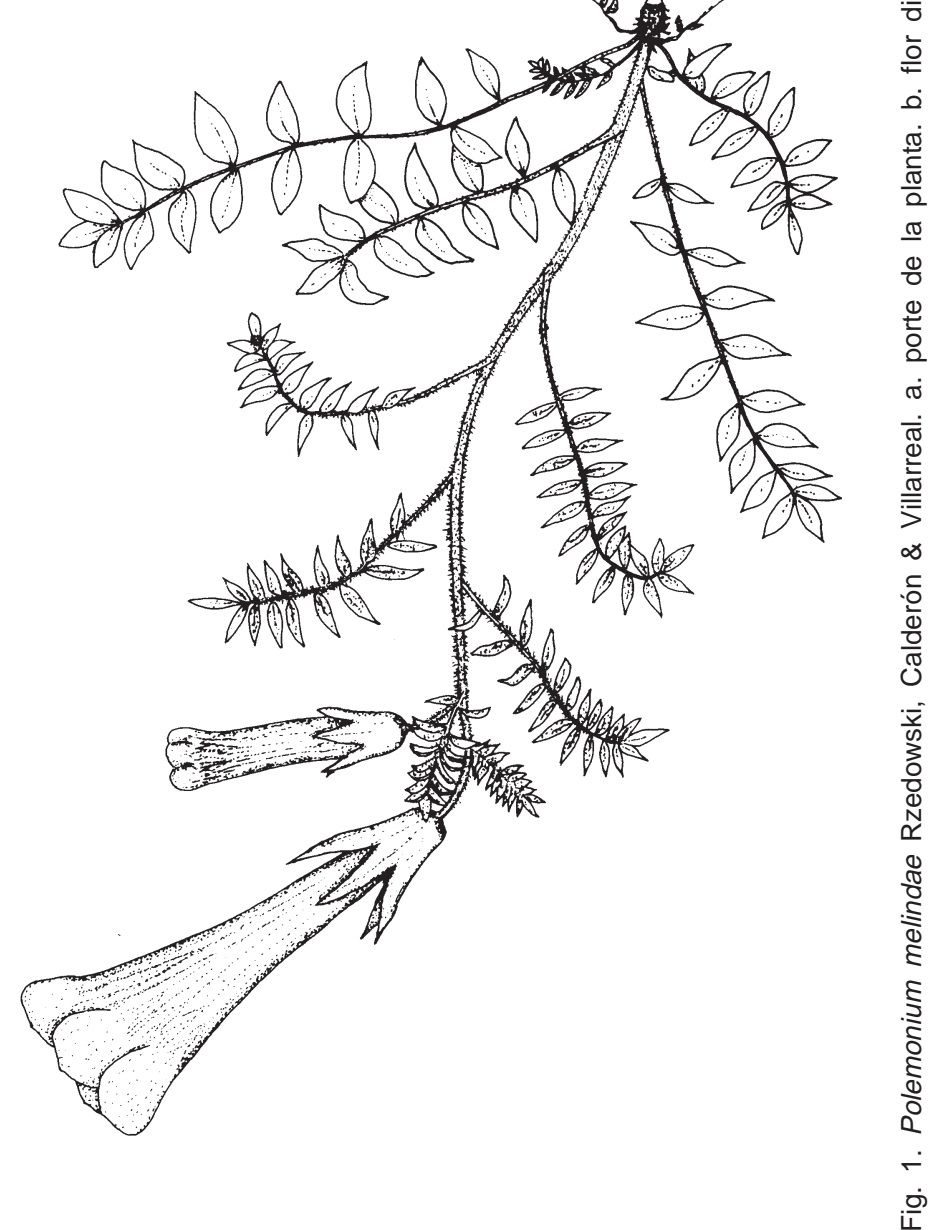
Planta herbácea perenne, rastrera o ascendente, hasta de $50 \mathrm{~cm}$ de largo; tallos más o menos profusamente ramificados, vilosos con pelos pluricelulares, variando de casi glabros a densamente lanosos; hojas imparipinnadas, peciolo de 10 a $35 \mathrm{~mm}$ de largo, raquis estrechamente alado, foliolos 15 a 27, alternos u opuestos, sésiles, lanceolados 0 a veces elípticos, de 7 a $25 \mathrm{~mm}$ de largo, de 2 a $6 \mathrm{~mm}$ de ancho, agudos en el ápice, cuneados a redondeados y los laterales más o menos oblicuos en la base, las bases de los tres foliolos superiores a menudo confluentes, densamente viloso-pilosos a casi glabros en ambas caras; flores dispuestas en la porción distal de las ramas, por lo general solitarias o por pares, a veces en grupos de tres, la primera en posición terminal, la segunda y la tercera (de presentarse) en la axilas de las hojas inmediatas, pedúnculos comunmente de 1 a $4 \mathrm{~cm}$, pero en ocasiones hasta de $15 \mathrm{~cm}$ de largo, con pubescencia similar a la de los tallos; cáliz tubular a angostamente campanulado, de 15 a 21(25) mm de largo, sus lóbulos angostamente triangulares y atenuados hacia un extremo redondeado en el ápice, a menudo no todos iguales entre sí, una a dos veces más largos que el tubo, viloso en el dorso; corola angostamente infundibuliforme, de color amarillo claro, a menudo tendiendo a verdoso, de 4.5 a $7 \mathrm{~cm}$ de largo, de (7)8 a $11 \mathrm{~mm}$ de diámetro a la mitad del largo del tubo, y de 1.5 a $3.5 \mathrm{~cm}$ de diámetro hacia el ápice, glabra, los lóbulos suborbiculares, de aproximadamente un cuarto del largo total de la corola; estambres inclusos a exsertos, filamentos de largo desigual, insertos cerca de la base de la corola, anteras suborbiculares a oblongas, de ca. $2 \mathrm{~mm}$ de largo; estilo aproximadamente tan largo como la corola, a veces ligeramente exserto; cápsula subcilíndrica, de 9 a $10 \mathrm{~mm}$ de largo, glabra; semillas oblongas, algo comprimidas, de ca. $2.5 \mathrm{~mm}$ de largo, cafés, brillantes.

Tipo: México. Querétaro. Parte alta del Cerro Zamorano, municipio de Colón, bosque de Abies y claros adyacentes, alt. 3100 m. 10.V.1987. J. Rzedowski 43240 (IEB).

Material adicional examinado: México. Chihuahua. 3.3 miles south of Chorro de Agua (abandoned sawmill) on road to Sawmill El Cuervo, 28 $11^{\prime} 40^{\prime \prime} \mathrm{N}, 108^{\circ} 03^{\prime} 50^{\prime \prime}$ W, T. $R$. y $R$. K. Van Devender y P. S. Martin 87-89 (ANSM, ARIZ, ENCB); Rio Verde, on road from El Vergel to Cienega Prieta, D. S. Correll y H. S. Gentry 22910 (ENCB). Durango. Ojo de Agua, $20 \mathrm{~km}$ de La Escondida por camino a Los Charcos, municipio de Mezquital, M. González et al. 1754 (ANSM, CIDIIR, IEB). Guanajuato. El Aserradero, Ojo de Agua del Tepetate, municipio de Acámbaro, A. Rubio 746 (EBUM); El Moro, municipio de Acámbaro, A. Rubio 653 (EBUM); Parte alta del Cerro El Moro, Sierra de los Agustinos, municipio de Acámbaro, H. Díaz Barriga 3963 (IEB). Querétaro. 1.1 mi below summit of Cerro Zamorano, M. F. Denton 1976 (ENCB); parte alta del Cerro Zamorano, municipio de Colón, J. Rzedowski 44445 (IEB).

$P$. melindae se ha colectado en altitudes entre 2700 y 3200 m; principalmente en bosques de coníferas, florece de abril a septiembre y se ha encontrado en fruto maduro en septiembre y octubre. A pesar de su área de distribución relativamente extendida, se trata de una planta muy poco frecuente, más bien esporádica, aunque a menudo abundante en los sitios en que vive.

Varios ejemplares de $P$. melindae fueron identificados inicialmente como $P$. pauciflorum S. Wats. (tipo: C. G. Pringle 1558 (GH, foto en IEB)), especie descrita de 
Chihuahua, pero colectada también en Coahuila, Nuevo León, Arizona y Texas. Es probable que estos dos taxa estén intimamente relacionados entre sí, pues además de numerosos rasgos de las partes vegetativas, comparten el carácter de la corola notablemente angosta y alargada, que no se presenta en los demás miembros conocidos de Polemonium (Davidson, 1950).

$P$. melindae se distingue de $P$. pauciflorum en sus corolas más grandes, tanto en longitud, como sobre todo en anchura, así como en el color de las mismas que es amarillo claro y a veces con toques de morado en el primero, mientras que en el segundo es amarillo con algo de rojo. Una diferencia adicional se observa en el ancho de los lóbulos del cáliz, que son angostamente triangulares en $P$. melindae y linear-atenuados en $P$. pauciflorum.

Las dos especies parecen ser simpátricas en la parte central de la Sierra Madre Occidental de Chihuahua, pero otras porciones de sus áreas conocidas de distribución no son coincidentes.

El nombre de la planta honra la memoria de la recién y prematuramente desaparecida Dra. Melinda F. Denton, ameritada botánica norteamericana, a quien se deben importantes contribuciones al conocimiento de la flora de México. Melinda fue la primera en colectar este Polemonium dentro del área de la Flora del Bajío y de regiones adyacentes.

\section{LITERATURA CITADA}

Bentham, G. 1845. Polemoniaceae. In: DC. Prodr. 9: 302-322.

Brand, A. 1907. Polemoniacae. Pflanzenr. IV, 250: 1-203.

Davidson, J. F. 1950. The genus Polemonium (Tournefort) L. Univ. Calif. Publ. Bot. 23(5): 209-282.

Gibson, D. G. 1970. Polemoniaceae. In: Flora of Guatemala. Fieldiana: Bot. 24(9): 85-97.

Nash, D. L. 1979. Polemoniaceae. Flora de Veracruz 7: 1-27.

Standley, P. C. 1914. A revision of the genus Cobaea. Contr. U. S. Nat. Herb. 17: 448-458.

Standley, P. C. 1924. Polemoniaceae. In: Trees and shrubs of Mexico. Contr. U. S. Nat. Herb. 23(4): 1208-1213.

Wherry, E. T. 1966. Polemoniaceae. Flora of Texas 1: 283-321. 